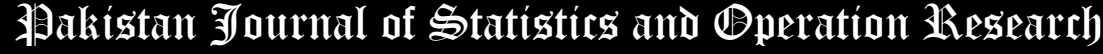

\section{A New Lifetime Model: Copulas, Properties and Real Lifetime Data Applications}

\author{
Wahid A. M. Shehata*
}

* Corresponding Author

Department of Mathematics, Statistics and Insurance, Faculty of Business, Ain Shams University, Egypt; wahid75maher@yahoo.com

\begin{abstract}
A new four parameter lifetime model called the Weibull generalized Lomax is proposed and studied. The new density function can be "right skewed", "symmetric" and "left skewed" and its corresponding failure rate function can be "monotonically decreasing", " monotonically increasing" and "constant". The skewness of the new distribution can negative and positive. The maximum likelihood method is employed and used for estimating the model parameters. Using the "biases" and "mean squared errors", we performed simulation experiments for assessing the finite sample behavior of the maximum likelihood estimators. The new model deserved to be chosen as the best model among many well-known Lomax extension such as exponentiated Lomax, gamma Lomax, Kumaraswamy Lomax, odd log-logistic Lomax, Macdonald Lomax, beta Lomax, reduced odd log-logistic Lomax, reduced Burr-Hatke Lomax, special generalized mixture Lomax and the standard Lomax distributions in modeling the "failure times" and the "service times" data sets.
\end{abstract}

Key Words: Lomax model; Simulations; Renyi's entropy copula; Farlie Gumbel Morgenstern copula; Simulation; Estimation; Modeling real data.

Mathematical Subject Classification: 62N01; 62N02; 62E10.

\section{Introduction}

The Lomax or Pareto type II distribution (Lomax (1954)), is a heavy-tail probabilistic model used in modeling business, actuarial science, biological sciences, engineering, economics, income and wealth inequality, queueing theory, size of cities and Internet traffic data sets. Harris (1968) and Atkinson and Harrison (1978) employed the Lomax (Lx) distribution in modeling data obtained from income and wealth. Corbellini et al. (2007) used the Lx distribution firm size data modeling. For applications in reliability and life testing experiments see Hassan Al-Ghamdi (2009). The Lx model is known as a special distribution form of Pearson system (type VI) and has also considered as a mixture of standard exponential (Exp) and standard gamma (Gam) distributions. The Lx model belongs to the family of "monotonically decreasing" hazard rate function (HRF) and considered as a limiting model of residual lifetimes at great age (see Balkema and de Hann (1974) and Chahkandi and Ganjali (2009)). The Lx distribution has been suggested as heavy tailed alternative model to the standard Exp, standard Weibull (W) and standard Gam distributions (see Bryson (1074)). For details about relation between the Lx model and the Burr XII and Compound Gamma (CGam) models see Tadikamalla (1980) and Durbey (1970).

The main aim of this work is to provide a flexible extension of the Lx distribution using the Weibull generalized-G (WG-G) family defined by Yousof et al. (2018). A random variable (RV) $Y$ has the Lomax (Lx) distribution with two parameters $\alpha$ and $\sigma$ if it has cumulative distribution function (CDF) (for $y>0$ ) given by

$$
G_{\alpha, \sigma}(y)=1-\left(1+\frac{y}{\sigma}\right)^{-\alpha}
$$


where $\alpha>0$ and $\sigma>0$ are the shape and scale parameters, respectively. Then the corresponding probability density function (PDF) of (1) is

$$
g_{\lambda, \sigma}(y)=\frac{\alpha}{\sigma}\left(1+\frac{y}{\sigma}\right)^{-(\alpha+1)} .
$$

Due to Yousof et al. (2018), the CDF of the WG-G family is given by

$$
F_{v, \gamma, \underline{\zeta}}(y)=1-\left.\exp \left\{-\left[\bar{G}_{\underline{\zeta}}(y)^{-\gamma}-1\right]^{v}\right\}\right|_{x \in R, v>0 \text { and } \gamma>0}
$$

where $\bar{G}_{\underline{\zeta}}(y)=1-G_{\underline{\zeta}}(y)$. The PDF corresponding to (3) is given by

$$
f_{v, \gamma, \underline{\zeta}}(y)=\left.v \gamma g_{\underline{\zeta}}(y) \frac{\left[1-\bar{G}_{\underline{\zeta}}(y)^{\gamma}\right]^{v-1}}{\bar{G}_{\underline{\zeta}}(y)^{v \gamma+1}} \exp \left\{-\left[\bar{G}_{\underline{\zeta}}(y)^{-\gamma}-1\right]^{v}\right\}\right|_{x \in R, v>0 \text { and } \gamma>0} \text {. }
$$

The Weibull generalized Lomax (WG-Lx) survival function (SF) is given by

$$
S_{\underline{\Psi}}(y)=\left.\exp \left\{-\left[\left(1+\frac{y}{\sigma}\right)^{\gamma \alpha}-1\right]^{v}\right\}\right|_{\left(x \in R^{+}\right.} \text {and } \underline{\left.\Psi=v, \gamma, \alpha, \sigma \in R^{+}\right)} \text {, }
$$

where $S_{\underline{\Psi}}(y)=1-\left.F_{\underline{\Psi}}(y)\right|_{(\underline{\Psi}=v, \gamma, \alpha, \sigma)}$. The PDF corresponding to (5) is given by

$$
f_{\underline{\Psi}}(y)=\left.v \gamma \frac{\alpha}{\sigma} \frac{\left[1-\left(1+\frac{y}{\sigma}\right)^{-\gamma \alpha}\right]^{v-1}}{\left(1+\frac{y}{\sigma}\right)^{(\gamma+1) \alpha+2}} \exp \left\{-\left[\left(1+\frac{y}{\sigma}\right)^{\gamma \alpha}-1\right]^{v}\right\}\right|_{\left(x \in R^{+} \text {and } v, \gamma, \alpha, \sigma \in R^{+}\right)} \text {. }
$$

For simulation of the WG-Lx model, we obtain the QF of $Y$ by inverting (5), say $y_{u}=F^{-1}(u)$, as

$$
y_{u}=\sigma\left\{1+[-\ln (1-u)]^{\frac{1}{v}}\right\}^{\frac{1}{\alpha \gamma}}-1,
$$

Equation (7) is used for simulating the new model. The HRF for the new model can be derived from $f_{\underline{\Psi}}(y) / k_{\underline{\Psi}}(y)$. Many useful Lx extensions can be found in Tahir et al. (2015) (the Weibull Lomax distribution), Cordeiro et al. (2018) (the one parameter Lomax system of densities), Altun et al. (2018a) (the odd log-logistic Lomax), Altun et al. (2018a) (Zografos-Balakrishnan Lomax distribution), Elbiely and Yousof (2018) (the Weibull generalized Lomax, Rayleigh generalized Lomax and exponential generalized Lomax distributions), Yousof et al. (2019) (the Topp Leone Poisson Lomax distribution), Goual and Yousof (2019) (the Lomax inverse Rayleigh), Gad et al. (2019) (the Burr type XII Lomax, the Lomax Burr type XII and the Lomax Lomax distributions), Yadav et al. (2020) (the Topp Leone Lomax distribution) and Ibrahim and Yousof (2020) (the Poisson Burr X generalized Lomax and Poisson Rayleigh generalized Lomax distributions). In this paper, we derive some new bivariate WG-Lx (BvWG-Lx) via Farlie Gumbel Morgenstern (FGM) Copula, modified Farlie Gumbel Morgenstern (FGM) Copula, Renyi's entropy and Clayton Copula. The Multivariate WG-Lx (MvWG-Lx) type is also presented using the Clayton Copula. However, future works could be allocated to study these new models. The concept of copulas is recently used by many authors such as Ibrahim et al. (2020) (Topp-Leone Lindley distribution), Al-babtain et al. (2020) (Marshall-Olkin binomialexponential distribution), Elgohari and Yousof (2020b) (Marshall-Olkin generalized-Weibull distribution), Salah et al. (2020) (odd burr Fréchet distribution), Yousof et al. (2021) (Xgamma reciprocal Rayleigh distribution), ElMorshedy et al. (2021) (the Poisson generalized exponential G family) and Ali et al. (2021a, b) (odd Burr generalized Rayleigh distribution and Marshall-Olkin Lehmann Burr type X distribution).

To illustrate the flexibility of the new PDF and its corresponding HRF we present Figure 1. Figure 1(a) gives some PDF plots for some selected parameters value. Figure 2(b) gives some HRF plots for some selected parameters value. Based on Figure 1(a) the WG-Lx density can be "right skewed", "symmetric", "left skewed" and "uniformed-PDF". Based on Figure 2(b) the WG-Lx HRF can be "monotonically decreasing" ( $v=\gamma=0.5, \alpha=1000, \sigma=10000)$, "monotonically increasing" ( $v=2.5, \gamma=2.5, \alpha=215, \sigma=1000) \&(v=1.25, \gamma=3000, \alpha=0.65, \sigma=8000)$ and "constant" ( $v=1, \gamma=1000, \alpha=0.5, \sigma=6000)$.

The WG-Lx model could be useful in statistical modeling in following cases:

1- The real-life data sets with " monotonically increasing HRF (asymmetric increasing HRF)".

2- The real-life data sets which have no extreme observations.

3- The real-life data sets which their nonparametric Kernel density estimation is bimodal and symmetric with right and left simple tail. 
The WG-Lx model proved its applicability against many well-known Lx extensions in following cases:

1- In statistical modeling of the failure times of the aircraft windshield observations, the WG-Lx model is better than many well-known Lx extension such as the McDonald Lx extension, the special generalized mixture Lx extension, the odd log-logistic Lx extension, the Gamma Lx extension, the Burr-Hatke Lx extension, the transmuted Topp-Leone Lx extension, the exponentiated Lx extension, the proportional reversed hazard rate Lx extension and the Kumaraswamy Lx extension under the Akaike-Information-Criteria, ConsistentInformation-Criteria, Bayesian-Information-Criteria and Hannan-Quinn-Information-Criteria.

2- In modeling the service times of the aircraft windshield, the WG-Lx model is better than many well-known Lx extension such as the McDonald Lx extension, the special generalized mixture Lx extension, the odd loglogistic Lx extension, the Gamma Lx extension, the Burr-Hatke Lx extension, the transmuted Topp-Leone Lx extension, the exponentiated Lx extension, the proportional reversed hazard rate Lx extension and the Kumaraswamy Lx extension under the Akaike-Information-Criteria, Consistent-Information-Criteria, Bayesian-Information-Criteria and Hannan-Quinn-Information-Criteria.
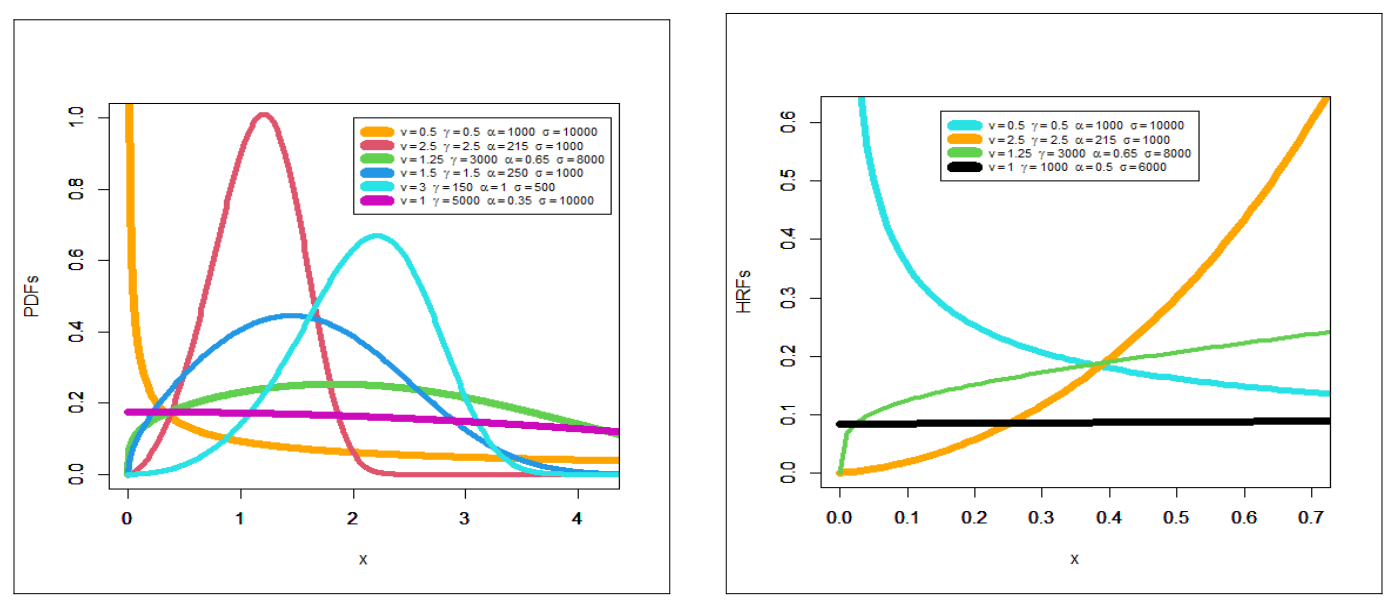

Figure 1: PDF and HRF plots for some selected parameters value.

\section{Copulas}

\section{BvWG-Lx type via FGM Copula}

Consider the joint CDF of the FGM family then

$$
\left.C_{r \in(-1,1)}(s, w)\right|_{r \in(-1,1)}=s w(1+r \overline{s w})
$$

where the marginal function $S=F_{1}\left(y_{1}\right), W=F_{2}\left(y_{2}\right)$ is a dependence parameter and for every $S, W \in(0,1)^{2}$, $C_{\gamma}(S, 0)=C_{\gamma}(0, W)=0$ which is "grounded minimum" and $C_{r}(S, 1)=S$ and $C_{r}(1, W)=W$ which is "grounded maximum". Then for $\varepsilon_{\left(y_{l}, \sigma_{l}\right)}=\left(1+\frac{y_{l}}{\sigma_{l}}\right)$, setting

and

$$
\bar{s}=\bar{s}_{\underline{\Psi}_{1}}=\left.\exp \left[-\left(d_{y_{1} ; \sigma_{1}}^{\gamma_{1} \alpha_{1}}-1\right)^{v_{1}}\right]\right|_{d_{y_{1} ; \sigma_{1}}=\left(1+\frac{y_{1}}{\sigma_{1}}\right)}
$$

then we have

$$
\bar{w}=\bar{w}_{\underline{\Psi}_{2}}=\left.\exp \left[-\left(d_{y_{2} ; \sigma_{2}}^{\gamma_{2} \alpha_{2}}-1\right)^{v_{2}}\right]\right|_{d_{y_{21} ; \sigma_{2}}=\left(1+\frac{y_{2}}{\sigma_{2}}\right)}
$$

$$
\begin{aligned}
& F_{r}\left(y_{1}, y_{2}\right)=C_{\gamma}\left(F_{\underline{\Psi}_{1}}\left(y_{1}\right), F_{\underline{\Psi}_{2}}\left(y_{2}\right)\right)=\left\{1-\exp \left[-\left(d_{y_{1} ; \sigma_{1}}^{\gamma_{1} \alpha_{1}}-1\right)^{v_{1}}\right]\right\} \\
& \times\left\{1-\exp \left[-\left(d_{y_{2} ; \sigma_{2}}^{\gamma_{2} \alpha_{2}}-1\right)^{v_{2}}\right]\right\}\left(1+\gamma\left\{\exp \left[-\left(d_{y_{1} ; \sigma_{1}}^{\gamma_{1} \alpha_{1}}-1\right)^{v_{1}}-\left(d_{y_{2} ; \sigma_{2}}^{\gamma_{2} \alpha_{2}}-1\right)^{v_{2}}\right]\right\}\right) .
\end{aligned}
$$

The joint PDF can then derived from $c_{\gamma}(s, w)=1+\left.r_{s}{ }^{*} w^{*}\right|_{\left(s^{*}=1-2 s\right.}$ and $\left.w^{*}=1-2 w\right)$.

\section{BvOBGR type via modified FGM Copula}

Consider the following modified version of the bivariate FGM copula defined as (see Rodriguez-Lallena and UbedaFlores (2004)):

$$
\left.C_{\gamma}(s, w)\right|_{\gamma \in[-1,1]}=s w[1+\gamma \varphi(s) \psi(w)]=s w+\gamma A(s) B(w),
$$


where $A(s)=s \varphi(s)$, and $B(w)=w \psi(w)$. Where $\varphi(s)$ and $\psi(w)$ are two absolutely continuous functions on (c), 10 with the following conditions:

1-The boundary condition: $\varphi(0)=\varphi(1)=\psi(0)=\psi(1)=0$.

2-Let

and

$$
\begin{aligned}
\tau_{1} & =\operatorname{lnf}\left\{\frac{\partial}{\partial s} A(s) \mid T_{1}(s)\right\}<0, \\
\tau_{2} & =\sup \left\{\frac{\partial}{\partial s} A(s) \mid T_{1}(s)\right\}<0, \\
\pi_{1} & =\operatorname{lnf}\left\{\frac{\partial}{\partial w} B(w) \mid T_{2}(w)\right\}>0,
\end{aligned}
$$

Then, $\operatorname{mln}\left(\tau_{1} \tau_{2}, \pi_{1} \pi_{2}\right) \geq 1$ where

$$
\pi_{2}=\sup \left\{\frac{\partial}{\partial w} B(w) \mid T_{2}(w)\right\}>0,
$$

$$
\begin{gathered}
\frac{\partial}{\partial s} A(s)=\varphi(s)+s \frac{\partial}{\partial s} \varphi(s) \\
T_{1}(s)=\left\{s: s \in(0,1) \mid \frac{\partial}{\partial s} A(s) \text { exists }\right\} \text { and } T_{2}(w)=\left\{w: w \in(0,1) \mid \frac{\partial}{\partial w} B(w) \text { exists }\right\} .
\end{gathered}
$$

\section{BvWG-Lx-FGM (Type I) model}

Here, we consider the following functional form for both $A(s)$ and $B(w)$ as

$$
C_{\gamma}(s, w)=\left\{1-\exp \left[-\left(d_{s ; \sigma_{1}}^{\gamma_{1} \alpha_{1}}-1\right)^{v_{1}}\right]\right\}\left\{1-\exp \left[-\left(d_{w ; \sigma_{2}}^{\gamma_{2} \alpha_{2}}-1\right)^{v_{2}}\right]\right\}+r A(s) B(w),
$$

where $A(s)=s \exp \left[-\left(d_{s ; \sigma_{1}}^{\gamma_{1} \alpha_{1}}-1\right)^{v_{1}}\right]$ and $B(w)=w \exp \left[-\left(d_{w ; \sigma_{2}}^{\gamma_{2} \alpha_{2}}-1\right)^{v_{2}}\right]$.

\section{BvWG-Lx-FGM (Type II) model}

Due to Mansour et al. (2020a) the CDF of the BvWG-Lx-FGM (Type II) model can be derived from

where

$$
C_{r}(s, w)=s F^{-1}(w)+w F^{-1}(s)-F^{-1}(s) F^{-1}(w),
$$

and

$$
1+F^{-1}(s)=\sigma_{1}\left\{1+[-\ln (1-s)]^{\frac{1}{v_{1}}}\right\}^{\frac{1}{\alpha_{1} \gamma_{1}}}
$$

\section{BvWG-Lx-FGM (Type III) model}

$$
1+F^{-1}(w)=\sigma_{2}\left\{1+[-\ln (1-w)]^{\frac{1}{v_{2}}}\right\}^{\frac{1}{\alpha_{2} \gamma_{2}}} .
$$

Consider the following functional form for both $\varphi(s)$ and $\psi(w)$ which satisfy all the conditions stated earlier where

$$
\left.\varphi(s)\right|_{\left(r_{1}>0\right)}=S^{r_{1}}(1-s)^{1-r_{1}} \text { and }\left.\psi(w)\right|_{\left(r_{2}>0\right)}=W^{r_{2}}(1-w)^{1-r_{2}} .
$$

The corresponding bivariate copula (henceforth, BvWG-Lx-FGM (Type III) copula) can be derived from

\section{BvWG-Lx-FGM (Type IV) model}

$$
C_{r, r_{1}, r_{2}}(s, w)=s w\left[1+r_{s}{ }^{r_{1}} w^{r_{2}}(1-s)^{1-r_{1}}(1-w)^{1-r_{2}}\right]
$$

Consider the following functional form for both $C(s)$ and $D(w)$ which satisfy all the conditions stated earlier where

$$
C(s)=s \log (1+\bar{s}) \text { and } D(w)=w \log (1+\bar{w}) .
$$

In this case, one can also derive a closed form expression for the associated CDF of the BvWG-Lx-FGM (Type IV) as

BvWG-Lx type via Renyi's entropy

$$
C_{\gamma}(S, W)=S W(1+r C(s) D(w)) .
$$

Consider theorem of Pougaza and Djafari (2011) where

$$
C(s, w)=y_{2} s+y_{1} w-y_{1} y_{2}
$$

where $S$ and $W$ are two absolutely continuous functions on $(0,1)$. Then, the associated BvWG-Lx will be

$$
C\left(y_{1}, y_{2}\right)=-y_{1} y_{2}+y_{2}\left\{1-\exp \left[-\left(d_{y_{1} ; \sigma_{1}}^{\gamma_{1} \alpha_{1}}-1\right)^{v_{1}}\right]\right\}+y_{1}\left\{1-\exp \left[-\left(d_{y_{2} ; \sigma_{2}}^{\gamma_{2} \alpha_{2}}-1\right)^{v_{2}}\right]\right\}
$$




\section{BvWG-Lx type via Clayton Copula}

The Clayton Copula can be considered as

$$
C_{\gamma}\left(w_{1}, w_{2}\right)=\left.\left(w_{1}^{-\gamma}+w_{2}^{-r}-1\right)^{-\frac{1}{r}}\right|_{\gamma \in[0, \infty]} .
$$

Let us assume that $Y \sim$ WG-Lx $\left(\underline{\Psi}_{1}\right)$ and $Z \sim$ WG-Lx $\left(\underline{\Psi}_{2}\right)$. Then, setting

and

$$
w_{1}=w\left(y \mid \underline{\Psi_{1}}\right)=1-\exp \left[-\left(d_{y ; \sigma_{1}}^{\gamma_{1} \alpha_{1}}-1\right)^{v_{1}}\right]
$$

Then, the BvWG-Lx type distribution can be derived as

$$
w_{2}=w\left(z \mid \underline{\Psi_{2}}\right)=1-\exp \left[-\left(d_{z ; \sigma_{2}}^{\gamma_{2} \alpha_{2}}-1\right)^{v_{2}}\right]
$$

$$
F_{\gamma}(y, z)=\left[\left\{1-\exp \left[-\left(d_{y ; \sigma_{1}}^{\gamma_{1} \alpha_{1}}-1\right)^{v_{1}}\right]\right\}^{-\gamma}+\left\{1-\exp \left[-\left(d_{z ; \sigma_{2}}^{\gamma_{2} \alpha_{2}}-1\right)^{v_{2}}\right]\right\}^{-\gamma}-1\right]^{-\frac{1}{\gamma}}
$$

\section{MvWG-Lx extension via Clayton Copula}

A straightforward $n$-dimensional extension from the above will be

Then, the MvWG-Lx extension can be expressed as

$$
C\left(w_{l}\right)=\left[\sum_{l=1}^{n} w_{l}^{-r}+1-n\right]^{-\frac{1}{r}}
$$

$$
C(\underline{Z})=\left(\sum_{l=1}^{n}\left\{1-\exp \left[-\left(d_{z ; \sigma_{2}}^{\gamma_{2} \alpha_{2}}-1\right)^{v_{2}}\right]\right\}^{-r}+1-n\right)^{-\frac{1}{r}}
$$

where $\underline{Z}=z_{1}, z_{2}, \cdots, z_{n}$.

\section{Mathematical properties}

\section{Useful representations}

Due to Yousof et al. (2018), the PDF in (6) can be expressed as

where

$$
f(y)=\sum_{k=0}^{\infty} \Delta_{k} g_{(1+k), \alpha, \sigma}(y)
$$

$$
\Delta_{k}=\sum_{l, j=0}^{\infty} \frac{(-1)^{1+l+j+k}}{\Gamma(1+l)}\left(\begin{array}{c}
l v \\
j
\end{array}\right)\left(\begin{array}{c}
\gamma(j-l v) \\
k
\end{array}\right)
$$

and $g_{(1+k), \alpha, \sigma}(y)$ is the PDF of the Lx model with power parameter $1+k$. By integrating Equation (8), the CDF of $Y$ becomes

$$
F(y)=\sum_{k=0}^{\infty} \Delta_{k} G_{(1+k), \alpha, \sigma}(y),
$$

where $G_{(1+k), \alpha, \sigma}(y)$ is the $\mathrm{CDF}$ of the Lx distribution with power parameter $1+k$.

\section{Moments and incomplete moments}

The $r^{t h}$ ordinary moment of $Y$ is given by $\mu_{r, Y}^{\prime}=E\left(y^{r}\right)=\int_{-\infty}^{\infty} y^{r} f(y) d y$, then we obtain

where

$$
\mu_{r, Y}^{\prime}=\left.\sum_{k=0}^{\infty} \sum_{d=0}^{r} \Delta_{k, d}^{(1+k, r)} B\left(1+k, 1+\frac{d-r}{\alpha}\right)\right|_{(\alpha>r)},
$$

and

$$
\Delta_{k, d}^{(1+k, r)}=\Delta_{k}(1+k) \sigma^{r}(-1)^{d}\left(\begin{array}{l}
r \\
d
\end{array}\right)
$$

$$
B\left(\nabla_{1}, \nabla_{2}\right)=\int_{0}^{1} s^{\nabla_{1}-1}(1-s)^{\nabla_{2}-1} d s
$$

Setting $r=1,2,3$ and 4 in (10), we have 


$$
\begin{aligned}
& E(Y)=\left.\sum_{k=0}^{\infty} \sum_{d=0}^{1} \Delta_{k, d}^{(1+k, 1)} B\left(1+k, \frac{d-1}{\alpha}+1\right)\right|_{(\alpha>1)}, \\
& E\left(Y^{2}\right)=\left.\sum_{k=0}^{\infty} \sum_{d=0}^{2} \Delta_{k, d}^{(1+k, 2)} B\left(1+k, \frac{d-2}{\alpha}+1\right)\right|_{(\alpha>2)}, \\
& E\left(Y^{3}\right)=\left.\sum_{k=0}^{\infty} \sum_{d=0}^{3} \Delta_{k, d}^{(1+k, 3)} B\left(1+k, \frac{d-3}{\alpha}+1\right)\right|_{(\alpha>3)},
\end{aligned}
$$

and

$$
E\left(Y^{4}\right)=\left.\sum_{k=0}^{\infty} \sum_{d=0}^{4} \Delta_{k, d}^{(1+k, 4)} B\left(1+k, \frac{d-4}{\alpha}+1\right)\right|_{(\alpha>4)},
$$

where $E(Y)=\mu_{1, Y}^{\prime}$ is the mean of $Y$. The $r^{t h}$ incomplete moment, say $I_{r, Y}(t)$, of $Y$ can be expressed, from (9) as

then

$$
I_{r, Y}(t)=\int_{-\infty}^{t} y^{r} f(y) d y=\sum_{k=0}^{\infty} \Delta_{k} \int_{-\infty}^{t} y^{r} g_{(1+k), \alpha, \sigma}(y) d y
$$

where

$$
I_{r, Y}(t)=\left.\sum_{k=0}^{\infty} \sum_{d=0}^{r} \Delta_{k, d}^{(1+k, r)} B_{t}\left(1+k, 1+\frac{d-r}{\alpha}\right)\right|_{(\alpha>r)},
$$

$$
B_{u}\left(\nabla_{1}, \nabla_{2}\right)=\int_{0}^{u} w^{\nabla_{1}-1}(1-w)^{\nabla_{2}-1} d w
$$

The first incomplete moment given by (11) with $r=1$ as

$$
I_{1, y}(t)=\left.\sum_{k=0}^{\infty} \sum_{d=0}^{1} \Delta_{k, d}^{(1+k, 1)} B_{t}\left(1+k, 1+\frac{d-1}{\alpha}\right)\right|_{(\alpha>1)} .
$$

The index of dispersion IxDis or the variance $\left(\mu_{2}\right)$ to mean ratio can derived as IxDis $(Y)=\mu_{2} / \mu_{1}^{\prime}$. It is a measure used to quantify whether a set of observed occurrences are clustered or dispersed compared to a standard statistical model.

\section{Numerical analysis}

By analyzing the $\mu_{1}^{\prime}, \mu_{2}$, skewness $\left(\beta_{1}\right)$, kurtosis $\left(\beta_{2}\right)$ and IxDis $(Y)$ numerically in Table 1 , it is noted that, the $\beta_{1}$ of the WG-Lx distribution can be negative and also positive. The spread for the $\beta_{2}$ of the WG-Lx model is ranging from -1129.85 to 311.698. The IxDis $(Y)$ for the WG-Lx model can be in $(0,1)$ and also $>1$ so it may be used as an "under-dispersed" and "over-dispersed" model.

Table 1: $\mu_{1}{ }^{\prime}, \mu_{2}, \beta_{1}, \beta_{2}$ and IxDis(Y) of the WG-Lx model.

\begin{tabular}{ccccccccc}
\hline $\mathrm{v}$ & $\gamma$ & $\alpha$ & $\sigma$ & $\mu_{1}{ }^{\prime}$ & $\mu_{2}$ & $\beta_{1}$ & $\beta_{2}$ & IxDis(Y) \\
\hline 0.5 & 2 & 5 & 15 & 1.115318 & 1.701461 & 1.582538 & 5.420254 & 1.5255390 \\
1 & & & & 0.935914 & 0.461627 & 0.813496 & 3.230566 & 0.4932369 \\
5 & & & & 1.000615 & 0.032425 & -0.5441214 & 3.28545 & 0.0324055 \\
10 & & & & 1.034504 & 0.009234 & -0.8243438 & 4.090645 & 0.0089257 \\
5 & 0.5 & 5 & 3 & 0.8871517 & 0.030056 & -0.4445040 & 3.114689 & 0.0338792 \\
& 1 & & & 0.4140281 & 0.005868 & -0.5105485 & 3.224178 & 0.0141719 \\
& 2 & & & 0.2001230 & 0.001297 & -0.5441214 & 3.285450 & 0.0064811 \\
& 3 & & & 0.1319302 & 0.000553 & -0.5553979 & 3.306883 & 0.0041941 \\
& 4 & & & 0.0983970 & 0.000305 & -0.5610533 & 3.317809 & 0.0030991 \\
& 5 & & & 0.0784549 & 0.000193 & -0.5644505 & -716.0823 & 0.0024573 \\
1.5 & 1.5 & 0.5 & 0.5 & 0.7048251 & 0.281039 & 1.3835870 & 5.710077 & 0.3987369 \\
& & 1 & & 0.2595549 & 0.025489 & 0.7938293 & 3.500659 & 0.0982025 \\
& & 2 & & 0.1130104 & 0.003996 & 0.5420847 & 2.916349 & 0.0353562 \\
& & 3 & & 0.0720898 & 0.001529 & 0.4630558 & 2.774388 & 0.0212056 \\
& & 4 & & 0.0529074 & 0.000799 & 0.4243545 & 3.268787 & 0.0150956 \\
& & 5 & & 0.0417835 & 0.000489 & 0.4013848 & 1.682906 & 0.0117068 \\
4 & 3 & 2 & 0.25 & 0.028029 & $4 \times 10^{-5}$ & 96.54233 & -1129.85 & 0.0014246
\end{tabular}




\begin{tabular}{cccccc}
0.5 & 0.056057 & 0.000159 & -0.3924604 & 311.698 & 0.0028493 \\
1 & 0.112115 & 0.000639 & -0.3925147 & 2.99214 & 0.0056985 \\
5 & 0.560573 & 0.015972 & -0.3924368 & 2.99139 & 0.0284925 \\
10 & 1.121145 & 0.063889 & -0.3924372 & 2.99139 & 0.0569851 \\
50 & 5.605726 & 1.597213 & -0.3924372 & 2.99130 & 0.2849253 \\
100 & 11.21145 & 6.388852 & -0.3924372 & 2.99139 & 0.5698506 \\
500 & 56.05726 & 159.7213 & -0.3924372 & 2.99139 & 2.8492530 \\
1000 & 112.1145 & 638.8852 & -0.3924372 & 2.99139 & 5.6985060 \\
\hline
\end{tabular}

\section{Some generating functions (GF)}

The moment generating function (MGF) can be derived using (8) as

$$
M_{Y}(t)=\sum_{k=0}^{\infty} \Delta_{k} M_{(1+k), \alpha, \sigma}(t)
$$

where $M_{(1+k), \alpha, \sigma}(t)$ is the MGF of the ExpLx model, then

where

$$
M_{Y}(t)=\left.\sum_{k, r=0}^{\infty} \sum_{d=0}^{r} \Delta_{k, d, r}^{(1+k, r)} B\left(1+k, 1+\frac{d-r}{\alpha}\right)\right|_{(\alpha>r)},
$$

$$
\Delta_{k, d, r}^{(1+k, r)}=\frac{t^{r}}{r !} \Delta_{k, d}^{(1+k, r)}
$$

The first $r$ derivatives of $M_{Y}(t)$, with respect to $t$ at $t=0$, yield the first $r$ moments about the origin, i.e., $\mu_{r, Y}^{\prime}=$ $E\left(Y^{r}\right)=\left.\frac{d^{r}}{d t^{r}} M_{Y}(t)\right|_{(t=0 \text { and } \mathrm{r}=1,2,3, \ldots)}$. The generating function GF (CGF) is the logarithm of the MGF. Thus, $r^{t h}$ cumulant, say $\kappa_{r}$, can be obtained from

$$
\kappa_{r}=\left.\frac{d^{r}}{d t^{r}} \log \left[\sum_{k, r=0}^{\infty} \sum_{d=0}^{r} \Delta_{k, d, r}^{(1+k, r)} B\left(1+k, 1+\frac{d-r}{\alpha}\right)\right]\right|_{(t=0, \text { and } \mathrm{r}=1,2,3, \ldots .)} .
$$

The $1^{\text {st }}$ cumulant is the mean $\left(\kappa_{1}=\mu_{1}^{\prime}\right)$, the $2^{\text {nd }}$ cumulant is the variance, and the $3^{\text {rd }}$ cumulant is the same as the $3^{\text {rd }}$ central moment $\kappa_{3}=\mu_{3}$. But $4^{\text {th }}$ and higher order cumulants are not equal to central moments. In some cases, theoretical treatments of problems in terms of cumulants are simpler than those using moments. When two or more RVs are statistically independent, the $r^{\text {th }}$ order cumulant of their sum is equal to the sum of their $r^{\text {th }}$ order cumulants. Moreover, the cumulants can be also obtained from

$$
\left.\kappa_{r}\right|_{r \geq 1}=\mu_{r}^{\prime}-\sum_{m=0}^{r-1}\left(\begin{array}{c}
r-1 \\
m-1
\end{array}\right) \mu_{r-m}^{\prime} \kappa_{m} .
$$

\section{Moment of the reversed residual}

The $n^{\text {th }}$ moment of the reversed residual life, say $V_{n}(t)=E\left[\left.(t-y)^{n}\right|_{y \leq t, t>0 \text { and } n=1,2, \ldots]}\right.$. We obtain

$$
V_{n}(t)=\frac{1}{F(t)} \int_{0}^{t}(t-y)^{n} d F(y)
$$

Then, the $n^{\text {th }}$ moment of the reversed residual life of $Y$ becomes

where

$$
V_{n}(t)=\left.\frac{1}{F(t)} \sum_{k=0}^{\infty} \sum_{d=0}^{r} \Delta_{k, d}^{(1+k, n) *} B_{t}\left(1+k, 1+\frac{d-r}{\alpha}\right)\right|_{(\alpha>r)},
$$

$$
\Delta_{k, d}^{(1+k, n) *}=\Delta_{k}(1+k) \sigma^{r}(-1)^{d}\left(\begin{array}{l}
r \\
d
\end{array}\right) \sum_{d=0}^{n}(-1)^{d}\left(\begin{array}{l}
n \\
d
\end{array}\right) t^{n-d}
$$

\section{Graphical assessment}

Graphically and using the biases and mean squared errors (MSEs), we can perform the simulation experiments to assess the finite sample behavior of the maximum likelihood estimations (MLEs). The assessment was based on $N$ $=1000$ replication for all $\left.n\right|_{(n=50,100, \ldots, 500)}$. The following algorithm is considered: 
I. Generate $N=1000$ samples of size $\left.n\right|_{(n=50,100, \ldots, 500)}$ from the WG-Lx distribution using (7);

II. Compute the MLEs for the $N=1000$ samples;

III. Compute the SEs of the MLEs for the 1000 samples. The standard errors (SEs) were computed by inverting the observed information matrix.

IV. Compute the biases and mean squared errors given for $\underline{\Psi}=v, \gamma, \alpha, \sigma$. We repeated these steps for $\left.n\right|_{(n=50,100, \ldots, 500)}$ with $v=1,2, . .100, \gamma=1,2, . .100, \alpha=1,2, . .100, \sigma=1,2, . .100$, so computing biases $\left(\operatorname{Bias}_{\underline{\underline{T}}}(n)\right)$, mean squared errors (MSEs) $\left(\operatorname{MSE}_{h}(n)\right)$ for $\underline{\Psi}=v, \gamma, \alpha, \sigma$ and $\left.n\right|_{(n=50,100, \ldots, 500)}$.

Figures 2, 3, 4 and 5 gives the biases (left panels) and MSEs (right panels) for the parameters $v, \gamma, \alpha$ and $\sigma$ respectively.

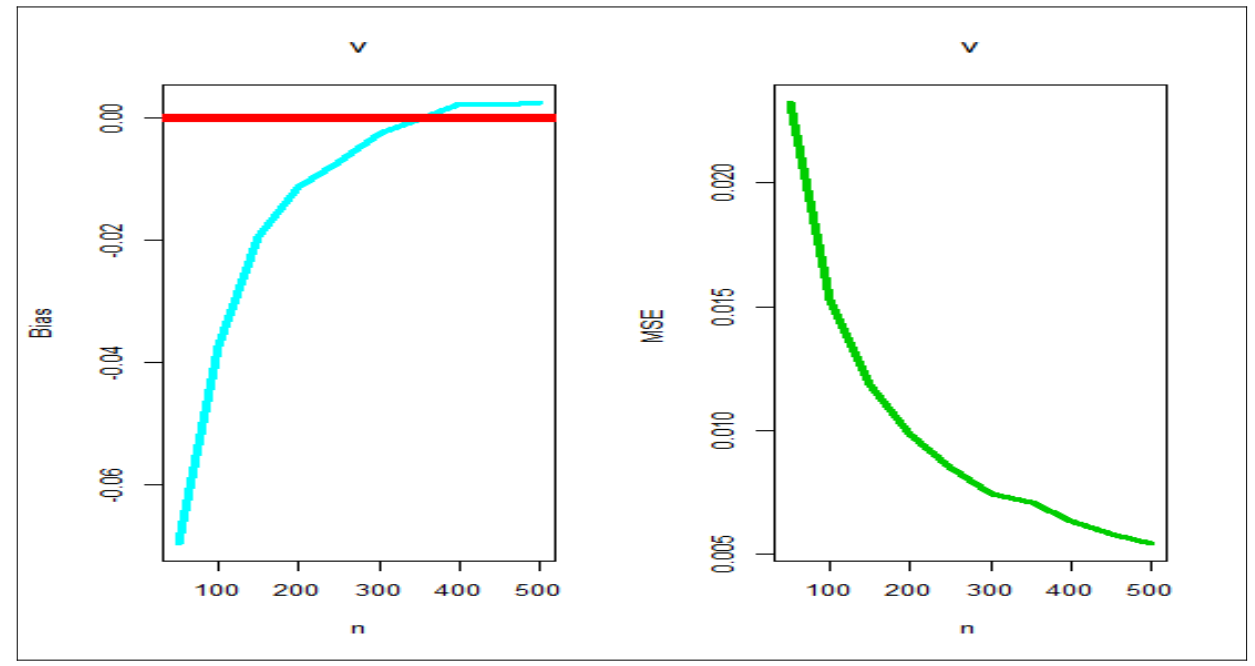

Figure 2: biases and mean squared errors for the parameter $v$.

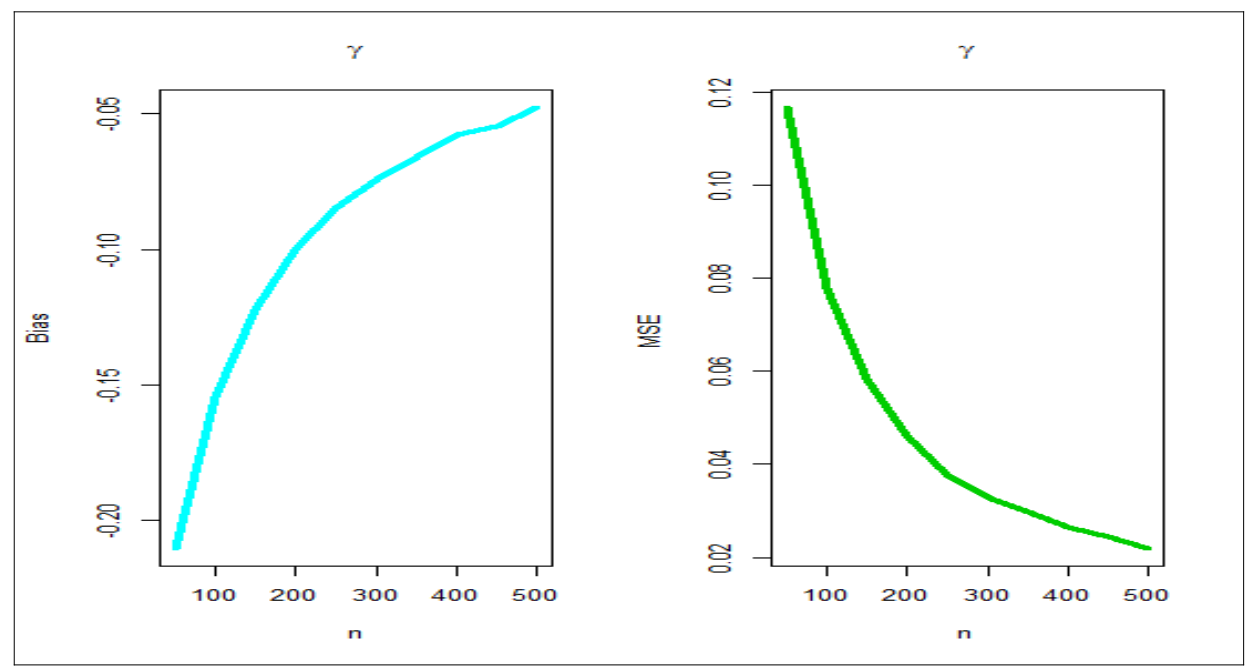

Figure 3: biases and mean squared errors for the parameter $\gamma$. 




Figure 4: biases and mean squared errors for the parameter $\alpha$.

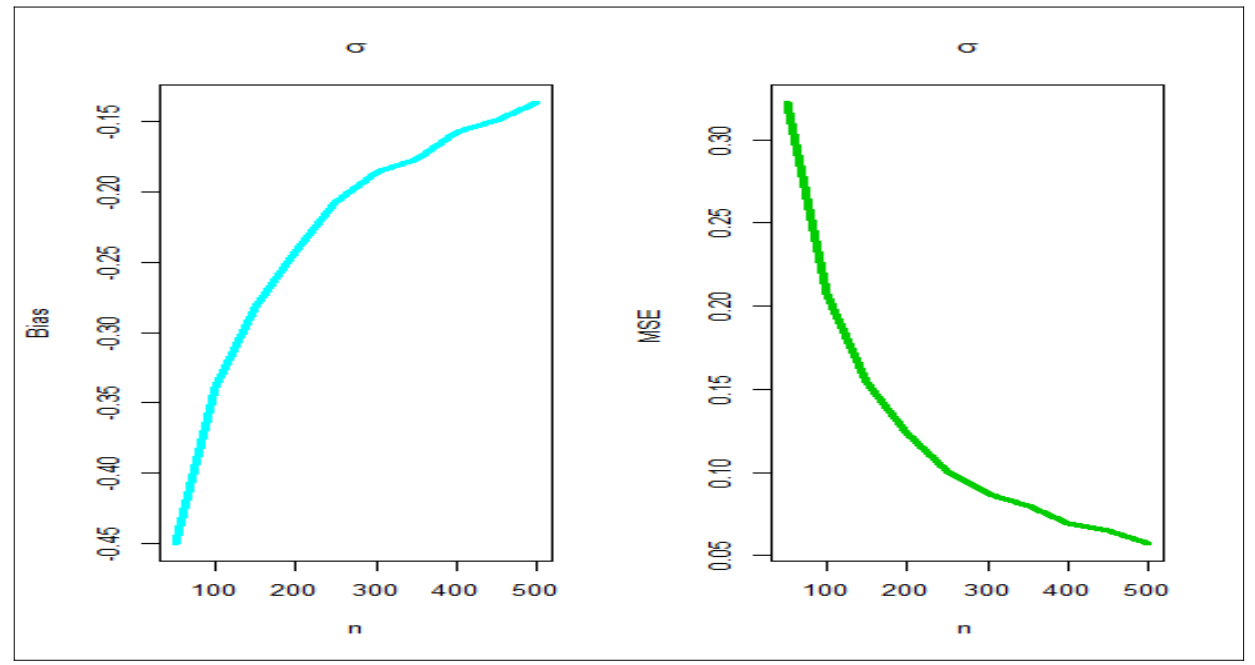

Figure 5: biases and mean squared errors for the parameter $\sigma$.

The left panels from show how the four biases vary with respect to $n$. The right panels show how the four MSEs vary with respect to $n$. The broken line in red in Figure 2 corresponds to the biases being 0 . From Figures 2-5 (left panels), the biases for each parameter are generally negative and tends to zero as $n \rightarrow \infty$. From Figures 25 (right panels), the MSEs for each parameter decrease to zero as $n \rightarrow \infty$.

\section{Applications}

In this section, we provide two real life applications to two real data sets to illustrate the importance and flexibility of the WG-Lx model. We compare the fit of the WG-Lx with some well-known competitive models (see Table 2)

Table 2: Competitive models.

\begin{tabular}{cccc}
\hline N. & Model & Abbreviation & Author \\
\hline 1 & Lomax & Lx & Lomax (1954) \\
2 & Exponentiated Lx & ExpLx & Gupta et al. (1998) \\
3 & Kumaraswamy Lx & KumLx & Lemonte and Cordeiro (2013) \\
4 & Macdonald Lx & McLx & Lemonte and Cordeiro (2013)
\end{tabular}




\begin{tabular}{cccc}
5 & Beta Lx & BLx & Lemonte and Cordeiro (2013) \\
6 & Gamma Lx & GamLx & Cordeiro et al. (2015) \\
7 & Transmuted Topp-Leone Lx & TTLLx & Yousof et al. (2017) \\
8 & Reduced TTLLx & RTTLLx & Yousof et al. (2017) \\
9 & Odd log-logistic Lx & OLLLx & Altun et al. (2018a) \\
10 & Reduced OLLLx & ROLLLx & Altun et al. (2018a) \\
11 & Reduced Burr-Hatke Lx & RBHLx & Yousof et al. (2018) \\
12 & Special generalized mixture Lomax & SGMLx & Chesneau and Yousof (2021) \\
13 & Reduced WG-Lx & RWG-Lx model & New \\
14 & Proportional reversed hazard rate Lx & PRHRLx & New \\
\hline
\end{tabular}

First data set: Failure times of 84 Aircraft Windshield: The first real data set (dataset I) represents the data on failure times of 84 aircraft windshield, this data is recently analyzed by El-Morshedy et al. (2021). The data are: 0.0400, 1.866, 2.3850, 3.443, 0.3010, 1.876, 2.4810, 3.4670, 0.3090, 1.8990, 2.610, 3.4780, 0.557, 1.9110, 2.625, 3.5780, $0.943,1.9120,2.632,3.5950,1.0700,1.914,2.6460,3.6990,1.1240,1.9810,2.661,3.7790,1.248,2.0100,2.688$, $3.9240,1.2810,2.0380,2.820,3,4.035,1.281,2.0850,2.890,4.121,1.3030,2.089,2.9020,4.167,1.4320,2.097$, $2.934,4.2400,1.480,2.1350,2.962,4.2550,1.505,2.1540,2.9640,4.2780,1.506,2.190,3.000,4.3050,1.568$, 2.1940, 3.103, 4.376, 1.615, 2.2230, 3.114, 4.449, 1.6190, 2.2240, 3.1170, 4.4850, 1.652, 2.2290, 3.166, 4.570, 1.652, $2.3000,3.344,4.602,1.7570,2.324,3.3760,4.6630$. Second data set: Service times of 63 Aircraft Windshield: The second real data set (dataset II) represents the data on service times of 63 aircraft windshield, this data is recently analyzed by El-Morshedy et al. (2021). The data are: 0.046, 1.436, 2.592, 0.140, 1.492, 2.600, 0.150, 1.580, 2.670, $0.248,1.7190,2.7170,0.2800,1.794,2.819,0.3130,1.915,2.820,0.389,1.9200,2.878,0.4870,1.9630,2.950,0.622$, $1.978,3.0030,0.9000,2.053,3.1020,0.9520,2.0650,3.3040,0.9960,2.1170,3.483,1.0030,2.1370,3.500,1.0100$, 2.141, 3.6220, 1.085, 2.1630, 3.6650, 1.092, 2.1830, 3.695, 1.1520, 2.2400, 4.015, 1.1830, 2.3410, 4.6280, 1.2440, $2.4350,4.8060,1.2490,2.4640,4.8810,1.2620,2.5430,5.1400$. Many other useful real life data sets can be found in Aryal et al. (2017), Yousof et al. (2018), Elbiely and Yousof (2018), Ibrahim and Yousof (2020), Yadav et al. (2020), Mansour et al. (2020e), Goual et al. (2020), Elgohari and Yousof (2020a) and Ibrahim et al. (2020). For exploring the extreme values, the box plot is plotted (see Figure 6). Based on Figure 6, we note that no extreme values were found in the two real life data sets. For checking the normality, the Quantile-Quantile (QQ) plot is sketched (see Figure 7). Based on Figures 7, we note that the normality is nearly exists. For exploring the HRF for real data, the total time test (TTT) plot is provided (see Figure 8). Based on Figure 8, we note that the HRF is "monotonically increasing" for the two real life data sets. For exploring the initial shape of real data nonparametrically, kernel density estimation (KDE) is provided (see Figure 9). Figure 9 show nonparametric KDE for exploring the data. Figures 10 and 11 give, Probability-Probability (P-P) plot (top left), estimated PDF (EPDF) (top right), estimated CDF (ECDF) (bottom left) and estimated HRF (EHRF) (bottom right) for data set I and II respectively.
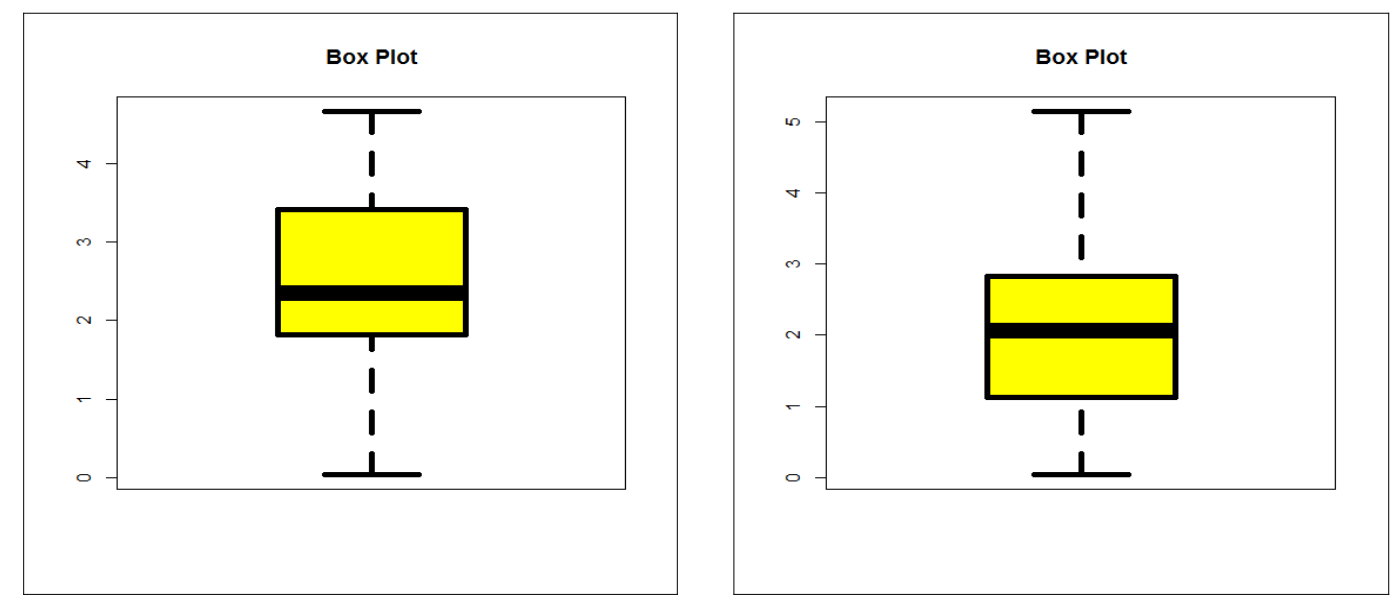

Figure 6: Box plots. 

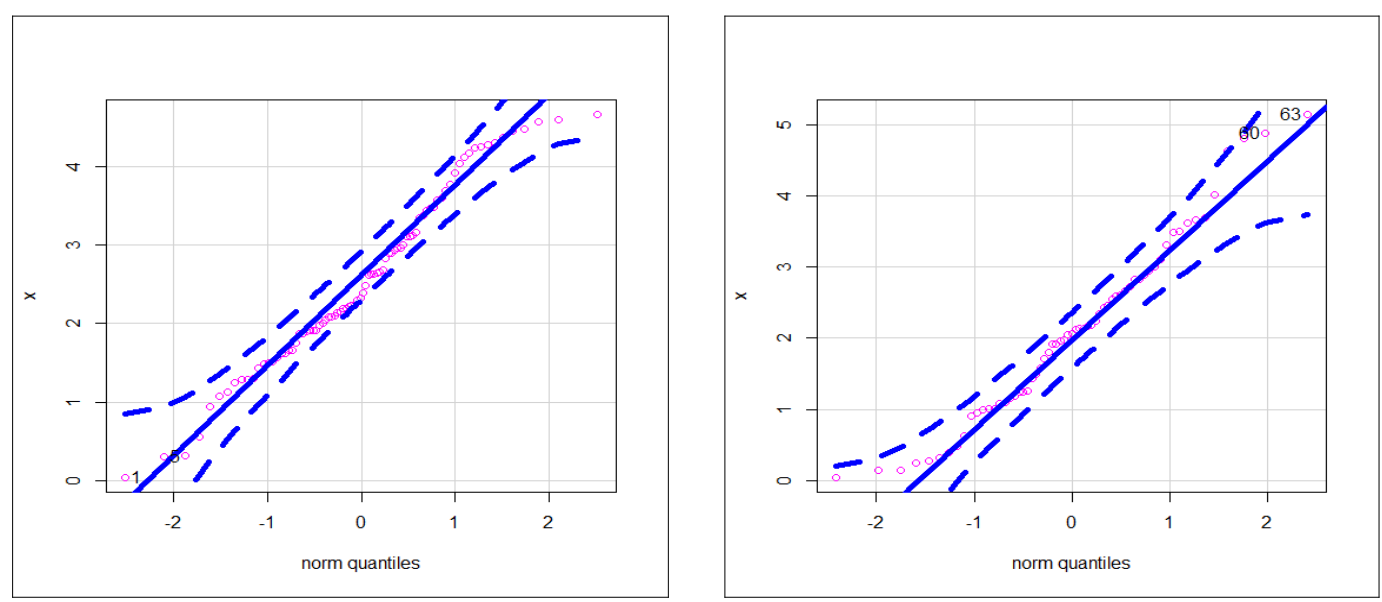

Figure 7: Normal QQ plots.
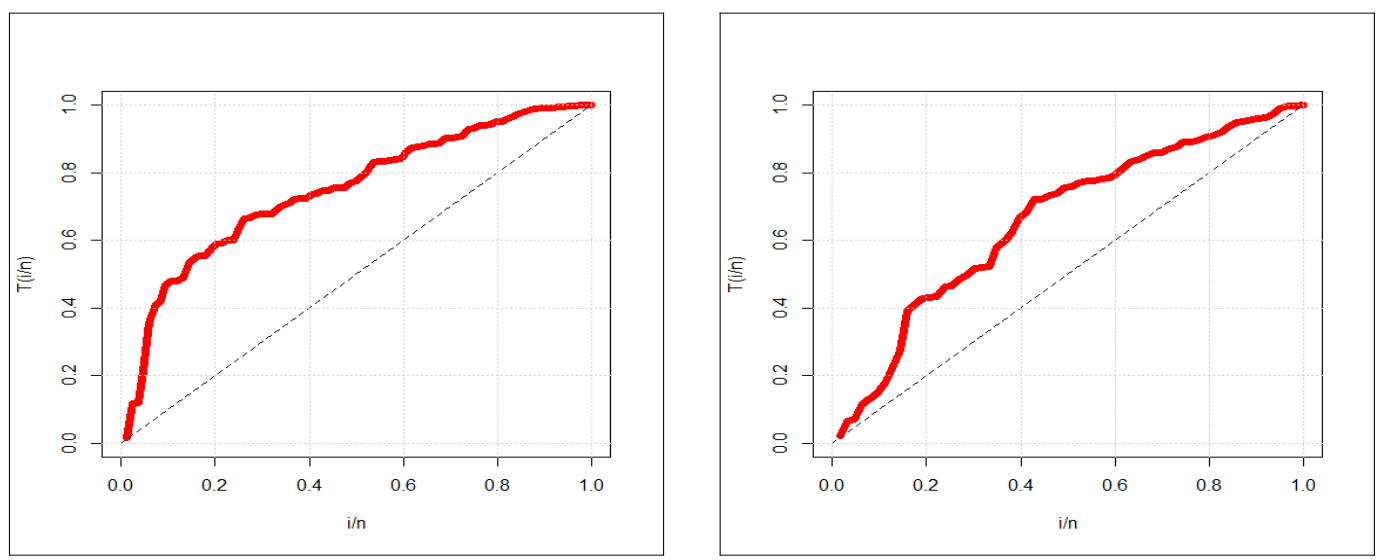

Figure 8: TTT plots.
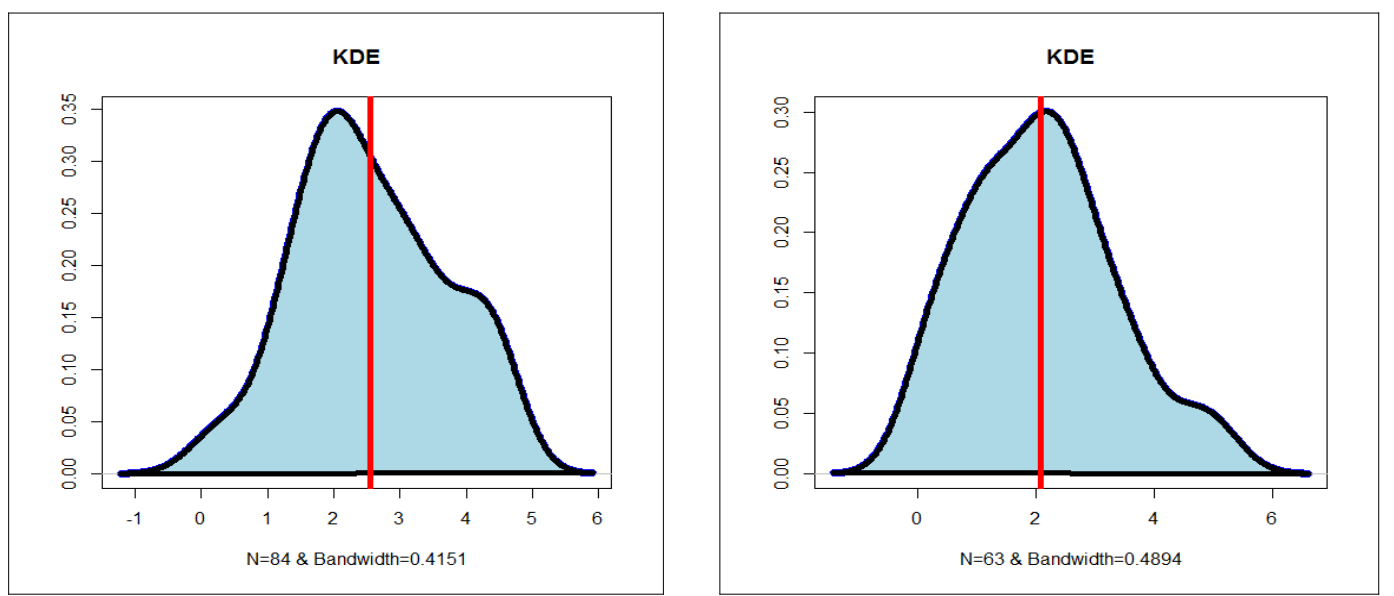

Figure 9: Nonparametric KDE. 
We estimate the unknown parameters of each model by maximum likelihood using "L-BFGS-B" method and the goodness-of-fit statistics Akaike information criterion (AIC), Bayesian IC (BIC), Consistent AIC (CAIC), HannanQuinn IC (HQIC), Anderson-Darling $\left(A^{*}\right)$ and Cramér-von Mises $\left(C^{*}\right)$ are used to compare the five models. For failure times data: the analysis results of are listed in Tables 3 and 4. Table 3 gives the MLEs and standard errors (SEs) for failure times data. Table 4 gives the $-\hat{\ell}$ and goodness-of-fits statistics for failure times data. For service times data: the analysis results of are listed in Tables 5 and 6. Table 5 gives the MLEs and SEs for service times data. Table 6 give the $-\hat{\ell}$ and goodness-of-fits statistics for the service times data. Based on Tables 4 and 6 , we note that the WGLx model gives the lowest values for the AIC, CAIC, BIC, HQIC, $A^{*}$ and $C^{*}$ among all fitted models. Hence, it could be chosen as the best model under these criteria.

Table 3: MLEs and SEs for failure times data.

\begin{tabular}{|c|c|c|c|c|c|}
\hline \multicolumn{2}{|l|}{ Model } & \multicolumn{4}{|c|}{ Estimates } \\
\hline WG-Lx $(\mathrm{v}, \gamma, \alpha, \sigma)$ & $\begin{array}{l}1.790561 \\
(0.16317)\end{array}$ & $\begin{array}{c}0.55679 \\
(0.23738)\end{array}$ & $\begin{array}{l}402.4722 \\
(17.5762)\end{array}$ & $\begin{array}{l}947.204 \\
(11.871)\end{array}$ & \\
\hline $\operatorname{McLx}(\mathrm{v}, \gamma, \mathrm{c}, \alpha, \sigma)$ & $\begin{array}{c}2.1875 \\
(0.5211)\end{array}$ & $\begin{array}{l}119.1751 \\
(140.297)\end{array}$ & $\begin{array}{l}12.4171 \\
(20.845)\end{array}$ & $\begin{array}{c}19.9243 \\
(38.9601)\end{array}$ & $\begin{array}{c}75.6606 \\
(147.24)\end{array}$ \\
\hline $\operatorname{TTLLx}(\mathrm{v}, \gamma, \alpha, \sigma)$ & $\begin{array}{l}-0.80750 \\
(0.13960)\end{array}$ & $\begin{array}{c}2.47663 \\
(0.54176)\end{array}$ & $\begin{array}{l}(15608.2) \\
(1602.37)\end{array}$ & $\begin{array}{l}(38628.3) \\
(123.936)\end{array}$ & \\
\hline $\operatorname{KumLx}(v, \gamma, \alpha, \sigma)$ & $\begin{array}{c}2.6150 \\
(0.3842)\end{array}$ & $\begin{array}{l}100.2756 \\
(120.486)\end{array}$ & $\begin{array}{l}5.27710 \\
(9.8116)\end{array}$ & $\begin{array}{c}78.6774 \\
(186.005)\end{array}$ & \\
\hline $\operatorname{BLx}(v, \gamma, \alpha, \sigma)$ & $\begin{array}{l}3.60360 \\
(0.6187)\end{array}$ & $\begin{array}{l}33.63870 \\
(63.7145)\end{array}$ & $\begin{array}{l}4.830700 \\
(9.23820)\end{array}$ & $\begin{array}{l}118.8374 \\
(428.927)\end{array}$ & \\
\hline $\operatorname{PRHRLx}(\gamma, \alpha, \sigma)$ & $\begin{array}{l}3.73 \times 10^{6} \\
1.01 \times 10^{6}\end{array}$ & $\begin{array}{c}4.707 \times 10^{-1} \\
(0.00001)\end{array}$ & $\begin{array}{l}4.49 \times 10^{6} \\
37.14684\end{array}$ & & \\
\hline $\operatorname{RTTLLx}(\mathrm{v}, \gamma, \alpha)$ & $\begin{array}{l}-0.84732 \\
(0.10010)\end{array}$ & $\begin{array}{l}5.520572 \\
(1.18479)\end{array}$ & $\begin{array}{l}1.156782 \\
(0.09588)\end{array}$ & & \\
\hline $\operatorname{SGMLx}(v, \alpha, \sigma)$ & $\begin{array}{c}-1.04 \times 10^{-1} \\
(0.1233)\end{array}$ & $\begin{array}{l}9.83 \times 10^{6} \\
(4843.3)\end{array}$ & $\begin{array}{l}1.18 \times 10^{7} \\
(501.04)\end{array}$ & & \\
\hline RWG-Lx $(v, \gamma, \alpha)$ & $\begin{array}{c}3.00116 \\
(0.27521)\end{array}$ & $\begin{array}{l}0.667532 \\
(0.00321)\end{array}$ & $\begin{array}{l}0.77532 \\
(0.0093)\end{array}$ & & \\
\hline $\operatorname{OLLLx}(\mathrm{v}, \alpha, \sigma)$ & $\begin{array}{c}2.32636 \\
\left(2.14 \times 10^{-1}\right)\end{array}$ & $\begin{array}{l}\left(7.17 \times 10^{5}\right) \\
\left(1.19 \times 10^{4}\right)\end{array}$ & $\begin{array}{l}\left.2.34 \times 10^{6}\right) \\
\left(2.61 \times 10^{1}\right)\end{array}$ & & \\
\hline $\operatorname{GamLx}(v, \alpha, \sigma)$ & $\begin{array}{l}3.587600 \\
(0.50334)\end{array}$ & $\begin{array}{l}52001.49 \\
(7955.00)\end{array}$ & $\begin{array}{l}37029.66 \\
(81.1644)\end{array}$ & & \\
\hline $\operatorname{ExpLx}(v, \alpha, \sigma)$ & $\begin{array}{c}3.626101 \\
(0.6236)\end{array}$ & $\begin{array}{l}20074.51 \\
(2041.83)\end{array}$ & $\begin{array}{l}26257.68 \\
(99.7417)\end{array}$ & & \\
\hline $\operatorname{ROLLLx}(\mathrm{v}, \alpha)$ & $\begin{array}{l}3.890564 \\
(0.36524)\end{array}$ & $\begin{array}{l}0.573161 \\
(0.01946)\end{array}$ & & & \\
\hline $\operatorname{RBHLx}(\alpha, \sigma)$ & $\begin{array}{l}10801754 \\
(983309)\end{array}$ & $\begin{array}{l}51367189 \\
(232312)\end{array}$ & & & \\
\hline $\operatorname{Lx}(\alpha, \sigma)$ & $\begin{array}{l}51425.353 \\
(5933.492)\end{array}$ & $\begin{array}{l}131789.8 \\
(296.119)\end{array}$ & & & \\
\hline
\end{tabular}

Table 4: $\ell$ and goodness-of-fits statistics for failure times data.

\begin{tabular}{cccccccc}
\hline Model & $\ell$ & AIC & CAIC & BIC & HQIC & $A^{*}$ & $C^{*}$ \\
\hline WG-Lx & $\mathbf{- 1 2 7 . 8 9 5 0}$ & $\mathbf{2 6 3 . 7 8 9 9}$ & $\mathbf{2 6 4 . 2 9 6 2}$ & $\mathbf{2 7 3 . 5 1 3 2}$ & $\mathbf{2 6 7 . 6 9 8 6}$ & $\mathbf{0 . 5 5 9 9}$ & $\mathbf{0 . 0 6 9 6}$ \\
McLx & -129.8023 & 269.6045 & 270.3640 & 281.8178 & 274.5170 & 0.6672 & 0.0858 \\
RWG-Lx & -132.1993 & 270.3987 & 270.6987 & 277.6911 & 273.3302 & 0.7593 & 0.0772 \\
OLLLx & -134.4235 & 274.8470 & 275.1470 & 282.1394 & 277.7785 & 0.9407 & 0.1009 \\
TTLLx & -135.5710 & 279.1410 & 279.6464 & 288.8633 & 283.0487 & 1.1257 & 0.1270 \\
GamLx & -138.4042 & 282.8083 & 283.1046 & 290.1363 & 285.7559 & 1.3666 & 0.1618 \\
BLx & -138.7177 & 285.4354 & 285.9354 & 295.2062 & 289.3654 & 1.4084 & 0.1682 \\
ExpLx & -141.3997 & 288.7994 & 289.0957 & 296.1273 & 291.7469 & 1.7435 & 0.2194 \\
ROLLLx & -142.8452 & 289.6904 & 289.8385 & 294.5520 & 291.6447 & 1.9566 & 0.2554 \\
SGMLx & -143.0874 & 292.1747 & 292.4747 & 299.4672 & 295.1062 & 1.3467 & 0.1578
\end{tabular}




\begin{tabular}{cccccccc} 
RTTLLX & -153.9810 & 313.9618 & 314.2618 & 321.2542 & 316.8933 & 3.7527 & 0.5592 \\
PRHRLx & -162.8771 & 331.7543 & 332.0542 & 339.0464 & 334.6855 & 1.3672 & 0.1609 \\
Lx & -164.9884 & 333.9767 & 334.1230 & 338.8620 & 335.9417 & 1.3976 & 0.1666 \\
RBHLx & -168.6041 & 341.2082 & 341.3562 & 346.0697 & 343.1624 & 1.6711 & 0.2069 \\
\hline
\end{tabular}
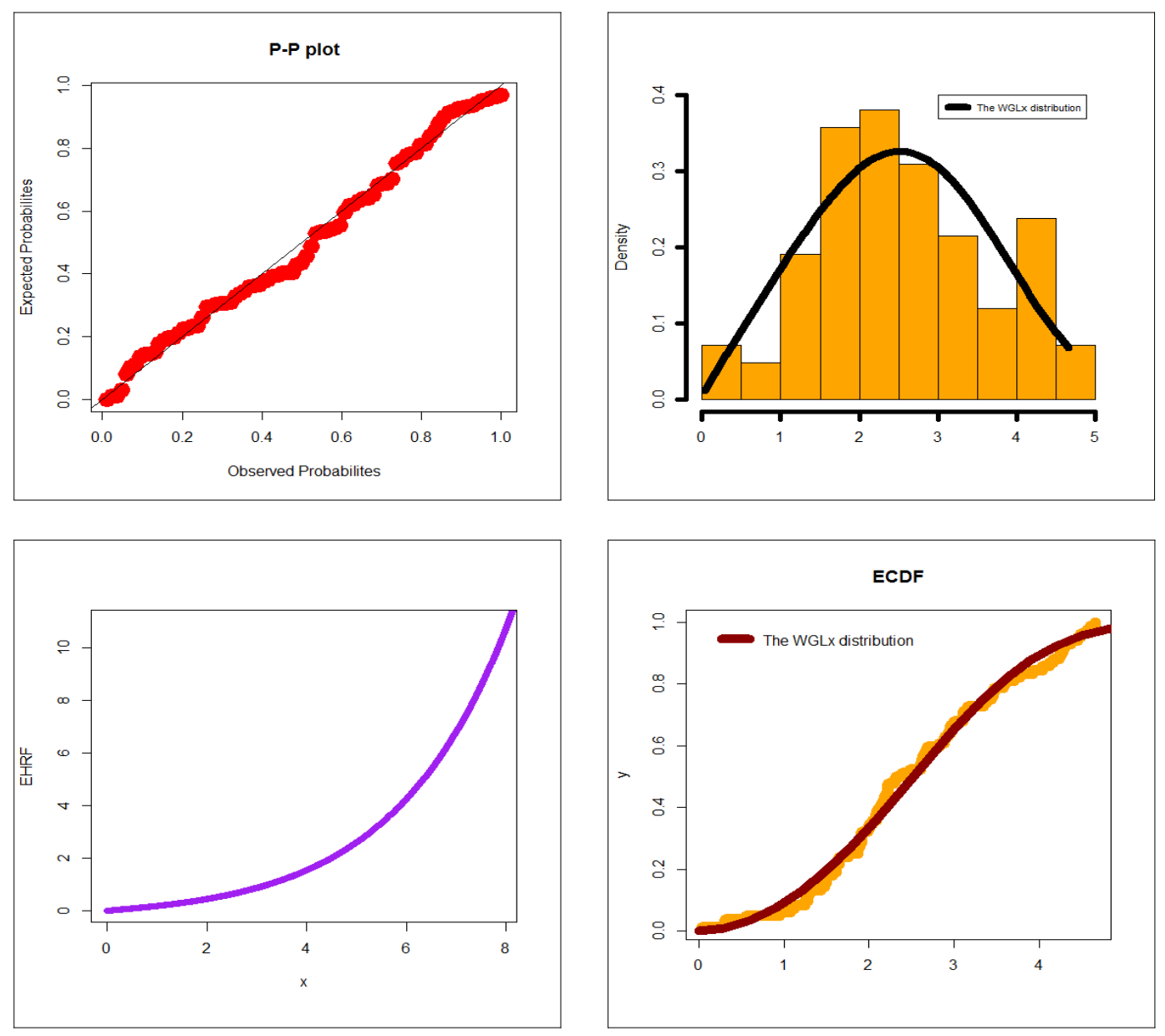

Figure 10: P-P plot, EPDF, ECDF and EHRF for data set $\mathbf{I}$.

Table 5: MLEs and SEs for service times data.

\begin{tabular}{ccccc}
\hline Model & \multicolumn{4}{c}{ Estimates } \\
\hline WG-Lx $(\mathrm{v}, \gamma, \alpha, \sigma)$ & $\mathbf{1 . 2 2 0 7 0}$ & $\mathbf{3 6 4 2 . 7 8 4}$ & $\mathbf{0 . 5 3 0 9 8}$ & $\mathbf{6 7 3 2 . 6 1}$ \\
& $\mathbf{( 0 . 1 3 1 8 9})$ & $\mathbf{( 3 2 . 1 7 6 9 )}$ & $\mathbf{( 0 . 3 2 0 1 )}$ & $\mathbf{( 8 . 1 7 6 4 )}$ \\
KumLx$(\mathrm{v}, \gamma, \alpha, \sigma)$ & 1.669132 & 60.5673 & 2.56490 & 65.06400 \\
& $(0.25701)$ & $(86.0131)$ & $(4.7589)$ & $(177.592)$ \\
$\operatorname{BLx}(\mathrm{v}, \gamma, \alpha, \sigma)$ & 1.9218 & 31.25943 & 4.968432 & 169.5719 \\
& $(0.3184)$ & $(316.841)$ & $(50.528)$ & $(339.207)$ \\
TTLLx $(\mathrm{v}, \gamma, \alpha, \sigma)$ & $(-0.6070)$ & 1.785780 & 2123.391 & 4822.789 \\
& $(0.21371)$ & $(0.41522)$ & $(163.915)$ & $(200.009)$ \\
PRHRLx $(\gamma, \alpha, \sigma)$ & $1.59 \times 10^{6}$ & $3.93 \times 10^{-1}$ & $1.30 \times 10^{6}$ & \\
& $2.01 \times 10^{3}$ & $0.0004 \times 10^{-1}$ & $0.95 \times 10^{6}$ & \\
RTTLLx $(\mathrm{v}, \gamma, \alpha)$ & -0.671451 & 2.74496 & 1.012381 & \\
& $(0.18746)$ & $(0.6696)$ & $(0.11405)$ & \\
SGMLx $(\mathrm{v}, \alpha, \sigma)$ & $-1.04 \times 10^{-1}$ & $6.451 \times 10^{6}$ & $6.33 \times 10^{6}$ &
\end{tabular}




\begin{tabular}{cccc} 
& $\left(4.1 \times 10^{-10}\right)$ & $\left(3.21 \times 10^{6}\right)$ & $(3.8573)$ \\
$\operatorname{RWG}-\operatorname{Lx}(\mathrm{v}, \gamma, \alpha)$ & 1.92707 & 1.349822 & 0.43660 \\
& $(0.21096)$ & $(12.6473)$ & $(4.09087)$ \\
$\operatorname{OLLLx}(\mathrm{v}, \alpha, \sigma)$ & 1.66419 & $6.340 \times 10^{5}$ & $2.01 \times 10^{6}$ \\
& $\left(1.79 \times 10^{-1}\right)$ & $\left(1.68 \times 10^{4}\right)$ & $7.22 \times 10^{6}$ \\
$\operatorname{GamLx}(\mathrm{v}, \alpha, \sigma)$ & 1.907334 & 35842.433 & 39197.57 \\
& $(0.3213)$ & $(6945.074)$ & $(151.653)$ \\
$\operatorname{ExpLx}(\mathrm{v}, \alpha, \sigma)$ & 1.91454 & 22971.154 & 32881.99 \\
& $(0.34822)$ & $(3209.533)$ & $(162.230)$ \\
$\operatorname{ROLLLx}(\mathrm{v}, \alpha)$ & 2.37233 & 0.6910933 & \\
& $(0.26825)$ & $(0.04488)$ & \\
$\operatorname{RBHLx}(\alpha, \sigma)$ & 14055522 & 53203423 & \\
& $(422.005)$ & $(28.52323)$ & \\
$\operatorname{Lx}(\alpha, \sigma)$ & 99269.78 & 207019.37 & \\
& $(11863.5)$ & $(301.2366)$ & \\
\hline
\end{tabular}

Table 6: $\ell$ and goodness-of-fits statistics for the service times data.

\begin{tabular}{cccccccc}
\hline Model & $\ell$ & AIC & CAIC & BIC & HQIC & $A^{*}$ & $C^{*}$ \\
\hline WG-Lx & $\mathbf{- 9 8 . 4 0 0 2 2}$ & $\mathbf{2 0 4 . 8 0 0 4}$ & $\mathbf{2 0 5 . 4 9 0 1}$ & $\mathbf{2 1 3 . 3 7 3 0}$ & $\mathbf{2 0 8 . 1 7 2 1}$ & $\mathbf{0 . 3 2 7 0}$ & $\mathbf{0 . 0 5 2 2}$ \\
KumLx & -100.8676 & 209.7353 & 210.4249 & 218.3078 & 213.1069 & 0.7391 & 0.1219 \\
RWG-Lx & -101.8349 & 209.6698 & 210.0766 & 216.0992 & 212.1985 & 0.8836 & 0.1459 \\
TTLLx & -102.4498 & 212.8996 & 213.5892 & 221.4723 & 216.2713 & 0.9431 & 0.1555 \\
GamLx & -102.8332 & 211.6663 & 212.0731 & 218.0958 & 214.1951 & 1.1120 & 0.1836 \\
SGMLx & -102.8939 & 211.7881 & 212.1949 & 218.2175 & 214.3168 & 1.1135 & 0.1839 \\
BLx & -102.9612 & 213.9224 & 214.6119 & 222.4948 & 217.2939 & 1.1336 & 0.1873 \\
ExpLx & -103.5498 & 213.0995 & 213.5063 & 219.5289 & 215.6282 & 1.2332 & 0.2037 \\
OLLLx & -104.9042 & 215.8082 & 216.2151 & 222.2376 & 218.3369 & 0.9425 & 0.1545 \\
PRHRLx & -109.2985 & 224.5973 & 225.0044 & 231.0267 & 227.1265 & 1.1263 & 0.1862 \\
Lx & -109.2989 & 222.5976 & 222.7976 & 226.8839 & 224.2834 & 1.1265 & 0.1862 \\
ROLLLx & -110.7288 & 225.4573 & 225.6573 & 229.7436 & 227.1431 & 2.3473 & 0.3908 \\
RTTLLx & -112.1855 & 230.3711 & 230.7777 & 236.8004 & 232.8997 & 2.6876 & 0.4532 \\
RBHLx & -112.6010 & 229.2010 & 229.4010 & 233.4873 & 230.8869 & 1.3985 & 0.2318 \\
\hline & & & & & & &
\end{tabular}



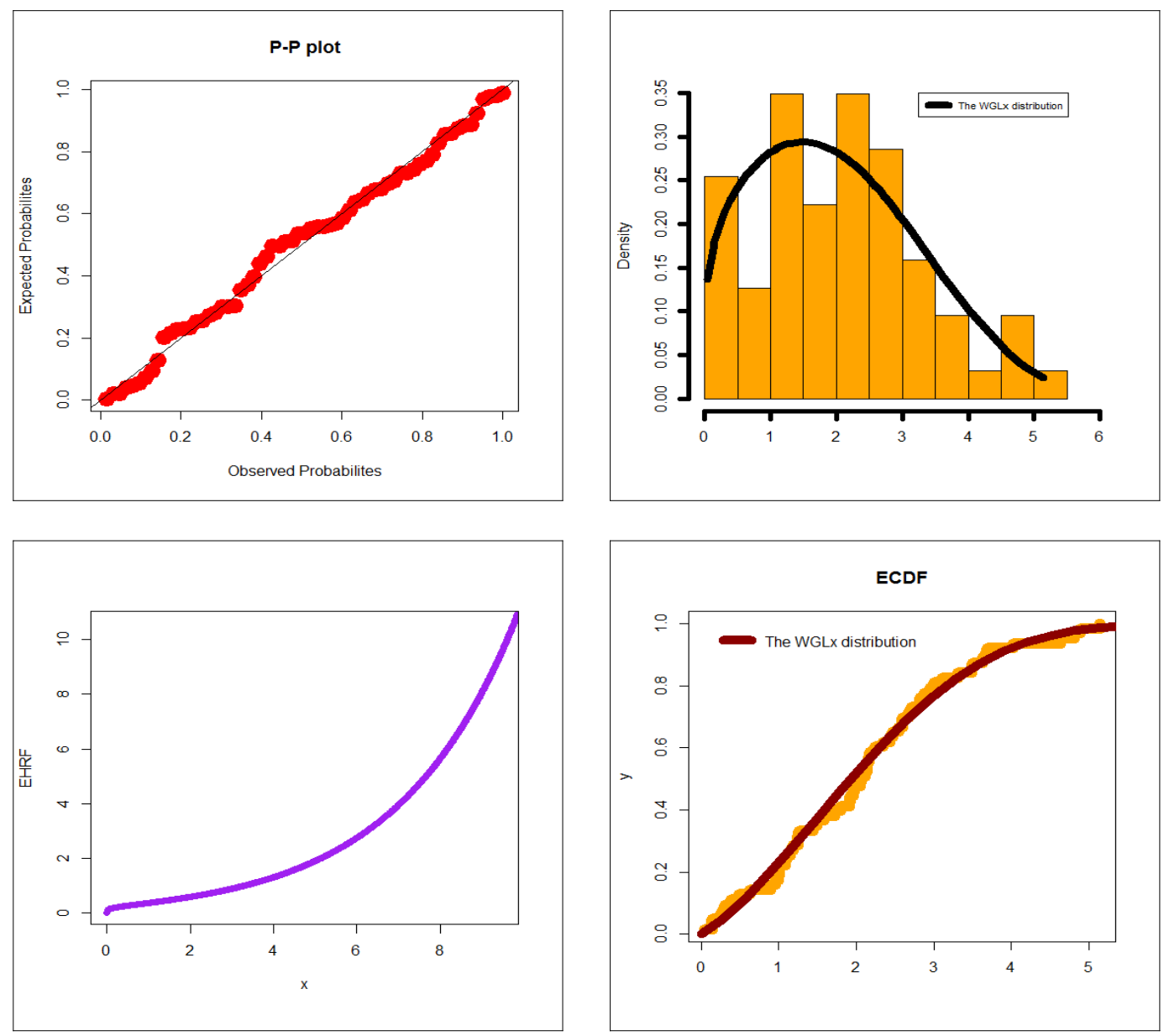

Figure 11: P-P plot, EPDF, ECDF and EHRF for data set II.

\section{Conclusions}

A new four parameter lifetime model called the Weibull generalized Lomax (WG-Lx) is proposed and studied. The WG-Lx density function can be "right skewed", "symmetric", "left skewed" and "uniformed density". The WG-Lx failure rate function can be "monotonically decreasing", " monotonically increasing" and "constant". The new WGLx density can be expressed as a mixture of the exponentiated Lomax model. The skewness of the WG-Lx distribution can negative and positive. The spread for the kurtosis of the WG-Lx model is ranging from - 1129.85 to 311.698. The index of dispersion for the WG-Lx model can be in $(0,1)$ and also $>1$ so it may be used as an "under-dispersed" and "over-dispersed" model. The maximum likelihood method is used to estimate the WG-Lx parameters. Using the "biases" and "mean squared errors", we performed simulation experiments for assessing the finite sample behavior of the maximum likelihood estimators. It is noted that, the biases for all parameters are generally negative and tends to 0 as $n \rightarrow \infty$ and the mean squared errors for all parameter decrease to 0 as $n \rightarrow \infty$. The WG-Lx model deserved to be chosen as the best model among many well-known Lomax extension such as exponentiated Lomax, gamma Lomax, Kumaraswamy Lomax, odd log-logistic Lomax, Macdonald Lomax, beta Lomax, reduced odd log-logistic Lomax, reduced Burr-Hatke Lomax, reduced WG-Lx, special generalized mixture Lomax and the standard Lomax distributions in modeling the "failure times" and the "service times" data sets. As a future potential work, the WG-Lx model can be validated using many new useful goodness-of-fit statistic tests in case of the right censored data sets such as the goodness-of-fit test of Nikulin-Rao-Robson (NRR) for right censored data, the modified NRR goodnessof-fit test for right censored data, the goodness-of-fit test of Bagdonavičius-Nikulin (BgN) for right censored data, modified $\mathrm{BgN}$ goodness-of-fit test for right censored data as recently performed by Ibrahim et al. (2019), Alizadeh et 
al. (2020), Goual et al. (2019, 2020), Mansour et al. (2020a-f), Yadav et al. (2020) and Goual and Yousof (2020), among others.

\section{References}

1. Aarset, M. V. (1987). How to identify a bathtub hazard rate. IEEE Transactions on Reliability, 36(1), 106-108.

2. Ali, M. M., Yousof, H. M. and Ibrahim, M. (2021a). A New Version of the Generalized Rayleigh Distribution with Copula, Properties, Applications and Different Methods of Estimation. In Optimal Decision Making in Operations Research \& Statistics: Methodologies and Applications, CRC Press. To appear.

3. Ali, M. M., Ibrahim, M. and Yousof, H. M. (2021b). Expanding the Burr X Model: Properties, Copula, Real Data Modeling and Different Methods of Estimation. In Optimal Decision Making in Operations Research \& Statistics: Methodologies and Applications, CRC Press. To appear.

4. Alizadeh, M., Yousof, H. M., Jahanshahi, S. M. A., Najibi, S. M. and Hamedani, G. G. (2020). The transmuted odd log-logistic-G family of distributions. Journal of Statistics and Management Systems, 23(4), 1-27.

5. Al-babtain, A. A., Elbatal, I. and Yousof, H. M. (2020). A New Flexible Three-Parameter Model: Properties, Clayton Copula, and Modeling Real Data. Symmetry, 12(3), 440.

6. Altun, E., Yousof, H. M. and Hamedani, G. G. (2018a). A new log-location regression model with influence diagnostics and residual analysis. Facta Universitatis, Series: Mathematics and Informatics, 33(3), 417-449.

7. Altun, E., Yousof, H. M., Chakraborty, S. and Handique, L. (2018b). Zografos-Balakrishnan Burr XII distribution: regression modeling and applications. International Journal of Mathematics and Statistics, 19(3), 46-70.

8. Aryal, G. R., Ortega, E. M., Hamedani, G. G. and Yousof, H. M. (2017). The Topp Leone Generated Weibull distribution: regression model, characterizations and applications, International Journal of Statistics and Probability, 6, 126-141.

9. Atkinson, A.B. and Harrison, A.J. (1978). Distribution of Personal Wealth in Britain (Cambridge University Press, Cambridge).

10. Balkema, A.A. and de Hann, L. Residual life at great age, Annals of Probability 2, 972-804, 1974.

11. Bryson, M. C. (1974). Heavy-tailed distribution: properties and tests, Technometrics 16, 161-68.

12. Chahkandi, M. and Ganjali, M. (2009). On some lifetime distributions with decrasing failure rate, Computational Statistics and Data Analysis 53, 4433-4440.

13. Chesneau, C. and Yousof, H. M. (2021). On a special generalized mixture class of probabilistic models. Journal of Nonlinear Modeling and Analysis, forthcoming.

14. Cordeiro, G. M., Yousof, H. M., Ramires, T. G. and Ortega, E. M. M. (2018). The Burr XII system of densities: properties, regression model and applications. Journal of Statistical Computation and Simulation, 88(3), 432-456.

15. Durbey, S. D. (1970). Compound gamma, beta and F distributions, Metrika 16, 27-31.

16. Gupta, R. C., Gupta, P. L. and Gupta, R. D. (1998). Modeling failure time data by Lehman alternatives. Communications in Statistics-Theory and methods, 27(4), 887-904.

17. Corbellini, A., Crosato, L., Ganugi, P and Mazzoli, M. (2007). Fitting Pareto II distributions on firm size: Statistical methodology and economic puzzles. Paper presented at the International Conference on Applied Stochastic Models and Data Analysis, Chania, Crete.

18. Cordeiro, G. M., Ortega, E. M. and Popovic, B. V. (2015). The gamma-Lomax distribution. Journal of Statistical computation and Simulation, 85(2), 305-319.

19. Elbiely, M. M. and Yousof, H. M. (2018). A New Extension of the Lomax Distribution and its Applications, Journal of Statistics and Applications, 2(1), 18-34.

20. Elgohari, H. and Yousof, H. M. (2020a). New Extension of Weibull Distribution: Copula, Mathematical Properties and Data Modeling. Statistics, Optimization \& Information Computing, 8(4), 972-993. https://doi.org/10.19139/soic-2310-5070-1036.

21. Elgohari, H. and Yousof, H. M. (2020b). New Extension of Weibull Distribution: Copula, Mathematical Properties and Data Modeling. Statistics, Optimization \& Information Computing, 8(4), 972-993. https://doi.org/10.19139/soic-2310-5070-1036

22. El-Morshedy, M., Alshammari, F. S., Hamed, Y. S., Eliwa, M. S., Yousof, H. M. (2021). A New Family of Continuous Probability Distributions. Entropy, 23, 194. https://doi.org/10.3390/e23020194

23. Gad, A. M., Hamedani, G. G., Salehabadi, S. M. and Yousof, H. M. (2019). The Burr XII-Burr XII distribution: mathematical properties and characterizations. Pakistan Journal of Statistics, 35(3), 229-248.

24. Gleaton, J. U. and Lynch, J.D. (2006). Properties of generalized loglogistic families of lifetime distributions. Journal of Probability and Statistical Science, 4, 51-64.

25. Goual, H. and Yousof, H. M. (2020). Validation of Burr XII inverse Rayleigh model via a modified chi-squared goodness-of-fit test. Journal of Applied Statistics, 47(3), 393-423.

26. Goual, H., Yousof, H. M. and Ali, M. M. (2019). Validation of the odd Lindley exponentiated exponential by a modified goodness of fit test with applications to censored and complete data. Pakistan Journal of Statistics and Operation Research, 15(3), 745-771.

27. Goual, H., Yousof, H. M., \& Ali, M. M. (2020). Lomax inverse Weibull model: properties, applications, and a modified Chisquared goodness-of-fit test for validation. Journal of Nonlinear Sciences \& Applications (JNSA), 13(6), 330-353.

28. Harris, C.M. (1968). The Pareto distribution as a queue service descipline, Operations Research, 16, 307-313.

29. Hassan, A.S. and Al-Ghamdi, A.S. (2009). Optimum step stress accelerated life testing for Lomax distibution, Journal of Applied Sciences Research, 5, 2153-2164.

30. Ibrahim, M., Altun, E. and Yousof, H. M. (2020). A new distribution for modeling lifetime data with different methods of estimation and censored regression modeling. Statistics, Optimization \& Information Computing, 8(2), 610-630.

31. Ibrahim, M. and Yousof, H. M. (2020). A new generalized Lomax model: statistical properties and applications, Journal of Data 
Science, 18(1), 190 - 217.

32. Ibrahim, M., Yadav, A. S., Yousof, H. M., Goual, H. and Hamedani, G. G. (2019). A new extension of Lindley distribution: modified validation test, characterizations and different methods of estimation. Communications for Statistical Applications and Methods, 26(5), 473-495.

33. Lemonte, A. J. and Cordeiro, G. M. (2013). An extended Lomax distribution. Statistics, 47(4), 800-816.

34. Lomax, K.S. (1954). Business failures: Another example of the analysis of failure data, Journal of the American Statistical Association, 49, 847-852.

35. Mansour, M. M., Ibrahim, M., Aidi, K., Shafique Butt, N., Ali, M. M., Yousof, H. M. and Hamed, M. S. (2020a). A New LogLogistic Lifetime Model with Mathematical Properties, Copula, Modified Goodness-of-Fit Test for Validation and Real Data Modeling. Mathematics, 8(9), 1508.

36. Mansour, M. M., Butt, N. S., Ansari, S. I., Yousof, H. M., Ali, M. M. and Ibrahim, M. (2020b). A new exponentiated Weibull distribution's extension: copula, mathematical properties and applications. Contributions to Mathematics, 1 (2020) 57-66. DOI: $10.47443 / \mathrm{cm} .2020 .0018$

37. Mansour, M., Korkmaz, M. Ç., Ali, M. M., Yousof, H. M., Ansari, S. I. and Ibrahim, M. (2020c). A generalization of the exponentiated Weibull model with properties, Copula and application. Eurasian Bulletin of Mathematics, 3(2), 84-102.

38. Mansour, M., Rasekhi, M., Ibrahim, M., Aidi, K., Yousof, H. M. and Elrazik, E. A. (2020d). A New Parametric Life Distribution with Modified Bagdonavičius-Nikulin Goodness-of-Fit Test for Censored Validation, Properties, Applications, and Different Estimation Methods. Entropy, 22(5), 592.

39. Mansour, M., Yousof, H. M., Shehata, W. A. and Ibrahim, M. (2020e). A new two parameter Burr XII distribution: properties, copula, different estimation methods and modeling acute bone cancer data. Journal of Nonlinear Science and Applications, 13(5), 223-238.

40. Mansour, M. M., Butt, N. S., Yousof, H. M., Ansari, S. I. and Ibrahim, M. (2020f). A Generalization of Reciprocal Exponential Model: Clayton Copula, Statistical Properties and Modeling Skewed and Symmetric Real Data Sets. Pakistan Journal of Statistics and Operation Research, 16(2), 373-386.

41. Pougaza, D. B. and Djafari, M. A. (2011). Maximum entropies copulas. Proceedings of the 30th international workshop on Bayesian inference and maximum Entropy methods in Science and Engineering, 329-336.

42. Rodriguez-Lallena, J. A. and Ubeda-Flores, M. (2004). A new class of bivariate copulas. Statistics and Probability Letters, 66, 31525.

43. Salah, M. M., El-Morshedy, M., Eliwa, M. S. and Yousof, H. M. (2020). Expanded Fréchet Model: Mathematical Properties, Copula, Different Estimation Methods, Applications and Validation Testing. Mathematics, 8(11), 1949.

44. Tadikamalla, P. R. (1980). A look at the Burr and realted distributions, International Statistical Review 48, $337-344$.

45. Yadav, A.S., Goual, H., Alotaibi, R.M. Rezk, H., Ali, M.M. and Yousof, H.M. (2020). Validation of the Topp-Leone-Lomax model via a modified Nikulin-Rao-Robson goodness-of-fit test with different methods of estimation. Symmetry, 12, 1-26. doi: $10.3390 /$ sym 12010057

46. Tahir, M. H., Mansoor, M., Cordeiro, G. M. and Zubair, M. (2015). The Weibull-Lomax distribution: properties and applications. Hacettepe Journal of Mathematics and Statistics, 44(2), 461-480.

47. Yousof, H. M., Ahsanullah, M. and Khalil, M. G. (2019). A New Zero-Truncated Version of the Poisson Burr XII Distribution: Characterizations and Properties. Journal of Statistical Theory and Applications, 18(1), 1-11.

48. Yousof, H. M., Alizadeh, M., Jahanshahiand, S. M. A., Ramires, T. G., Ghosh, I. and Hamedani, G. G. (2017). The transmuted Topp-Leone G family of distributions: theory, characterizations and applications. Journal of Data Science, 15(4), 723-740.

49. Yousof, H. M., Altun, E., Ramires, T. G., Alizadeh, M. and Rasekhi, M. (2018). A new family of distributions with properties, regression models and applications, Journal of Statistics and Management Systems, 21, 1, 163-188.

50. Yousof, H. M., Majumder, M., Jahanshahi, S. M. A., Ali, M. M. and Hamedani G. G. (2018). A new Weibull class of distributions: theory, characterizations and applications, Journal of Statistical Research of Iran, 15, 45-83.

51. Yousof, H. M., Ali, M. M. Goual, H. and Ibrahim, M. (2021). A New Reciprocal Rayleigh Extension: Properties, Copulas, Different Methods of Estimation and Modified Right Censored Test for Validation. Statistics in Transition. To appear. 